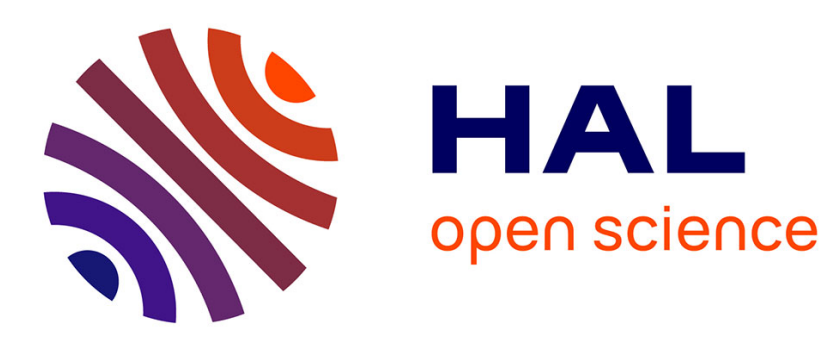

\title{
Performance of two Low-Rank STAP Filters in a Heterogeneous Noise
}

Guillaume Ginolhac, Philippe Forster, Frédéric Pascal, Jean-Philippe Ovarlez

\section{To cite this version:}

Guillaume Ginolhac, Philippe Forster, Frédéric Pascal, Jean-Philippe Ovarlez. Performance of two Low-Rank STAP Filters in a Heterogeneous Noise. IEEE Transactions on Signal Processing, 2013, 61 (1), pp.57 - 61. 10.1109/TSP.2012.2226162 . hal-00828338

\section{HAL Id: hal-00828338 https://hal.science/hal-00828338}

Submitted on 30 May 2013

HAL is a multi-disciplinary open access archive for the deposit and dissemination of scientific research documents, whether they are published or not. The documents may come from teaching and research institutions in France or abroad, or from public or private research centers.
L'archive ouverte pluridisciplinaire HAL, est destinée au dépôt et à la diffusion de documents scientifiques de niveau recherche, publiés ou non, émanant des établissements d'enseignement et de recherche français ou étrangers, des laboratoires publics ou privés. 


\title{
Performance of two Low-Rank STAP Filters in a Heterogeneous Noise
}

\author{
Guillaume Ginolhac, Philippe Forster, Member, IEEE, \\ Frédéric Pascal, Member, IEEE and Jean-Philippe Ovarlez
}

\begin{abstract}
This paper considers the Space Time Adaptive Processing (STAP) problem where the disturbance is modeled as the sum of a Low-Rank (LR) Spherically Invariant Random Vector (SIRV) clutter and a zero-mean white Gaussian noise. To derive our adaptive LR-STAP filters, the estimation of the projector onto the clutter subspace is performed from the Sample Covariance Matrix (SCM) and the Normalized Sample Covariance Matrix (NSCM). We compute the theoretical performance of both corresponding LR-STAP filters through the analysis of the Signal to Interference plus Noise Ratio (SINR) Loss, based on a perturbation analysis. Numerical simulations validate the theoretical formula and allow to show that the LR-STAP filter built from the SCM performance does not depend on the heterogeneity of the SIRV clutter whereas the LR-STAP filter built from the NSCM performance does.
\end{abstract}

\section{Index Terms}

SIRV, Low-Rank Clutter, STAP, Normalized Sample Covariance Matrix, perturbation method.

\section{INTRODUCTION}

Space Time Adaptive Processing (STAP) is a technique used in airborne phased array radar to detect moving target embedded in an interference background such as jamming or strong clutter [1]. While conventional radars are capable of detecting targets both in the time domain related to target range and in the frequency domain related to target velocity, STAP uses an additional domain (space) related to the target angular localization. The consequence is a two-dimensional adaptive filtering technique which

Guillaume Ginolhac and Philippe Forster are with the SATIE - ENS Cachan, email: guillaume.ginolhac,philippe.forster@satie.ens-cachan.fr. Jean-Philippe Ovarlez and Frédéric Pascal are with SONDRA Supelec, email:ovarlez@onera.fr,frederic.pascal@supelec.fr 
uses jointly temporal and spatial dimensions to cancel interference and to improve target detection. In most works on radar, the clutter is assumed to be a simple Gaussian process. But firstly, the increase of the radar resolution leads to a higher scene heterogeneity where the clutter can be no longer modeled by a Gaussian process [2], [3]. To take this heterogeneity into account, one can use the Spherically Invariant Random Vector (SIRV) product model, first introduced by Yao [4] in the information theory community. This is a compound-Gaussian model, well-known for its good statistical properties and for its good correspondence to several real data sets [5], [6]. Secondly in side-looking STAP (as considered in this paper), the ground clutter can be shown to have a Low Rank (LR) structure from Brennan rule [7]. In this paper, the disturbance is then modeled as the sum of a LR-SIRV clutter and a zero-mean white Gaussian noise.

In practice, the disturbance covariance matrix is generally unknown and an estimate is required to perform the STAP processing. This estimation procedure requires the so-called secondary data which are assumed to be independent and share the same distribution as the observation under test. In a STAP framework, the dimension of the covariance matrix is important (number of sensors times number of pulses). Commonly, the number of secondary data has to be upper than two times this dimension to ensure the classical 3dB loss for the performance results [8]. One of the advantage of the LR techniques is that this rule can be strongly relaxed to preserve such a performance [9], [10], [11], [12]. Another problem in STAP comes from these secondary data which are often contaminated by the secondary lobes of the target under study or other targets with same angular and velocity properties. It is also possible to have outliers in the STAP data cube like for example in highway STAP data with cars traffic-jam or convoys tracking. In such cases, LR techniques exhibit another advantage on classical methods: for a quite low Signal-to-Clutter Ratio (SCR), LR techniques are robust to secondary data contamination, see e.g., [9], [13]. This robustness directly relies on the choice of the covariance matrix estimate, from which the projector estimate will be derived. It seems obvious that the well-known Sample Covariance Matrix (SCM) is not adapted to strong contamination problems since this estimate depends on the power of each data sample. Thus, it is interesting to consider other covariance matrix estimates, independent of the data power, i.e. self-normalized estimates. This is the case of the Normalized Sample Covariance Matrix (NSCM) [14]. We then proposed in [15] to build the clutter subspace projector on the NSCM. The corresponding LR-STAP filter exhibits interesting results: it is more robust to secondary data contamination by target components than the LR-STAP filter obtained from the SCM. Moreover, we showed in [16] that the projector onto the clutter subspace built from the NSCM is a consistent estimate of the true one when the disturbance is modeled as the sum of a LR-SIRV clutter and a white Gaussian 
noise.

Under the two hypotheses of Gaussian clutter plus noise and orthogonality of the target signal with respect to the clutter subspace, the theoretical analysis of LR-STAP filters has been conducted in the seminal works [9], [11], [12] (with SCM) and next in [17] (with NSCM). In this paper, we relax the first hypothesis and we consider the much more realistic case of a LR-SIRV clutter plus white Gaussian noise. However, for mathematical tractability the second hypothesis is kept. Our work studies the Signal to Interference plus Noise Ratio (SINR) Loss by means of a perturbation analysis [18]. Numerical simulations validate our results even in a case of non orthogonality of the target signal with respect to the clutter subspace and give a comparison between the filters performance.

The following convention is adopted: italic indicates a scalar quantity, lower case boldface indicates a vector quantity and upper case boldface a matrix. ${ }^{T}$ denotes the transpose operator and ${ }^{H}$ the transpose conjugate. $E[]$ is the expected value operator. $\mathscr{C} \mathscr{N}(\mathbf{a}, \mathbf{M})$ is a complex Gaussian vector of mean a and of covariance matrix $\mathbf{M} . \mathbf{I}_{m}$ is the $m \times m$-identity matrix. $\chi^{2}(n)$ is a Chi-square random variable with $n$ degrees of freedom.

\section{LOW-RANK STAP FILTERS}

In STAP [1], the radar receiver consists in an array of $N$ antenna elements processing $M$ pulses in a coherent processing interval. Let us set $m=N M$. In this framework, we receive a signal $\mathbf{x} \in \mathbb{C}^{m}$ consisting in a known complex signal $\mathbf{d}$ corrupted by an additive disturbance $\mathbf{n}$. We also have $K$ secondary data $\mathbf{x}_{k}$ which only contain the disturbance:

$$
\begin{aligned}
\mathbf{x} & =\alpha \mathbf{d}+\mathbf{n} \\
\mathbf{x}_{k} & =\mathbf{n}_{k} \quad k=1, \ldots, K
\end{aligned}
$$

where $\alpha$ is a deterministic complex attenuation and $\mathbf{d}$ has unit norm. We assume that $\mathbf{n}$ and $\mathbf{n}_{k}$ are independent and share the same statistical distribution. They are modeled as the sum of a clutter, $\mathbf{c}$ or $\mathbf{c}_{k}$, and a white Gaussian noise, $\mathbf{b}$ or $\mathbf{b}_{k}$. The processes $\mathbf{b}$ and $\mathbf{b}_{k}$ are modeled as zero-mean complex Gaussian noises $\mathscr{C} \mathscr{N}\left(\mathbf{0}, \lambda \mathbf{I}_{m}\right)$. Concerning the clutter $\mathbf{c}$ and $\mathbf{c}_{k}$, we consider that its power in each cell $k$ and in the cell under test is different. In such a situation, it is common to model this kind of clutter by a SIRV [19], [20], [21]. A SIRV [4] is a non-homogeneous Gaussian random vector with random power: its randomness is induced by spatial variation in the radar backscattering. Therefore, $\mathbf{c}$ (resp. $\mathbf{c}_{k}$ ) is the product of a positive random variable $\tau$ (resp. $\tau_{k}$ ), called the texture, and a $m$-dimensional independent 
complex Gaussian vector $\mathscr{C} \mathscr{N}(\mathbf{0}, \mathbf{C}) \mathbf{g}\left(\right.$ resp. $\left.\mathbf{g}_{k}\right)$, called the speckle:

$$
\begin{aligned}
\mathbf{c} & =\sqrt{\tau} \mathbf{g} \\
\mathbf{c}_{k} & =\sqrt{\tau} \mathbf{g}_{k} \quad k=1, \ldots, K
\end{aligned}
$$

In side-looking STAP, we are able to evaluate the clutter rank thanks to Brennan rule [7] which leads to a low rank structure for the STAP clutter $\mathbf{c}$ and $\mathbf{c}_{k}$, i.e. $\operatorname{rank}(\mathbf{C})=r \ll m$. Let $\lambda_{1}>\lambda_{2}>\ldots>\lambda_{r}>$ $0=\ldots=0$ and $\left\{\mathbf{u}_{1}, \ldots, \mathbf{u}_{r}, \mathbf{u}_{r+1}, \ldots, \mathbf{u}_{m}\right\}$ be the eigensystem of $\mathbf{C}$. The covariance matrix of $\mathbf{n}$ and $\mathbf{n}_{k}$ is then given by:

$$
\boldsymbol{\Sigma}=E(\tau) \mathbf{C}+\lambda \mathbf{I}_{m}=\mathbf{S}_{\Sigma}+\lambda \mathbf{I}_{m}
$$

with corresponding eigensystem $E(\tau) \lambda_{1}+\lambda>E(\tau) \lambda_{2}+\lambda>\ldots>E(\tau) \lambda_{r}+\lambda>\lambda=\ldots=\lambda$ and $\left\{\mathbf{u}_{1}, \ldots, \mathbf{u}_{r}, \mathbf{u}_{r+1}, \ldots, \mathbf{u}_{m}\right\}$. We define the projector onto the clutter subspace $\boldsymbol{\Pi}_{c}$ and the projector onto the orthogonal of the clutter subspace $\Pi_{c}^{\perp}=\mathbf{I}_{m}-\boldsymbol{\Pi}_{c}$ [9], [11]:

$$
\boldsymbol{\Pi}_{c}^{\perp}=\mathbf{I}_{m}-\sum_{i=1}^{r} \mathbf{u}_{i} \mathbf{u}_{i}^{H} .
$$

The optimal STAP filter $\mathbf{w}_{\text {opt }}$ is [1]:

$$
\mathbf{w}_{\text {opt }}=\boldsymbol{\Sigma}^{-1} \mathbf{d}
$$

In the low-rank assumption, this optimal filter is approximated by [9], [11]: $\mathbf{w}_{l r-o p t} \approx \mathbf{\Pi}_{c}^{\perp} \mathbf{d}$.

In practical cases, since the covariance matrix $\Sigma$ (and therefore also $\Pi_{c}^{\perp}$ ) is unknown, it is necessary to estimate them from the secondary data $\left\{\mathbf{x}_{k}\right\}_{k \in(1, K)}$. The common way to estimate the projector $\boldsymbol{\Pi}_{c}^{\perp}$ is based on the Sample Covariance Matrix (SCM). From its Eigenvalue Decomposition (EVD), we obtain the estimated projector onto the clutter subspace [9], [11]:

$$
\hat{\mathbf{\Pi}}_{c S C M}^{\perp}=\mathbf{I}_{m}-\sum_{i=1}^{r} \hat{\mathbf{u}}_{i} \hat{\mathbf{u}}_{i}^{H}
$$

For the case of SIRV clutter without white Gaussian noise, it is well known that the NSCM [14] allows to reach better detection performances than the SCM. Therefore, we proposed in [15] to build $\boldsymbol{\Pi}_{c}$ from the NSCM. Indeed, even if the NSCM is a biased estimate of the covariance matrix $\boldsymbol{\Sigma}$, we showed in [16] that the associated projector estimate is consistent when the disturbance is a LR-SIRV clutter plus a white Gaussian noise. Moreover, the corresponding LR-STAP filter allows to reach good robustness to data contamination [15]. The NSCM is defined as:

$$
\hat{\mathbf{R}}_{N S C M}=\frac{m}{K} \sum_{k=1}^{K} \frac{\mathbf{x}_{k} \mathbf{x}_{k}^{H}}{\mathbf{x}_{k}^{H} \mathbf{x}_{k}} .
$$


From its EVD, we obtain another estimated projector onto the clutter subspace [9], [11]:

$$
\hat{\boldsymbol{\Pi}}_{c N S C M}^{\perp}=\mathbf{I}_{m}-\sum_{i=1}^{r} \hat{\mathbf{u}}_{i}^{\prime} \hat{\mathbf{u}}_{i}^{\prime H} .
$$

The two sub-optimal STAP filters $\hat{\mathbf{w}}$ studied in this paper are:

$$
\begin{aligned}
\hat{\mathbf{w}}_{S C M} & =\hat{\boldsymbol{\Pi}}_{c S C M}^{\perp} \mathbf{d} \\
\hat{\mathbf{w}}_{N S C M} & =\hat{\boldsymbol{\Pi}}_{c N S C M}^{\perp} \mathbf{d}
\end{aligned} .
$$

\section{THEORETICAL SINR LOSS}

As in previous works on LR-STAP theoretical performance analysis [11], the following usual assumption is made for mathematical tractability: the projection of the steering vector on the true interference subspace is negligible, i.e. $\mathbf{u}_{i}^{H} \mathbf{d} \approx 0$ for $i=1, \ldots, r$. This just means that the target is not fully embedded in the clutter ridge. We will check in next section by simulations that the theoretical result is also valid even in a case of non orthogonality of the target signal with respect to the clutter subspace. From the structure of $\boldsymbol{\Sigma}$, we have the following relations:

$$
\boldsymbol{\Sigma} \mathbf{d}=\lambda \mathbf{d} \quad, \quad \boldsymbol{\Sigma}^{-1} \mathbf{d}=\frac{1}{\lambda} \mathbf{d} \quad \text { and } \quad \boldsymbol{\Pi}_{c}^{\perp} \mathbf{d}=\mathbf{d} .
$$

The generic STAP filter output is $\mathbf{y}=\mathbf{w}^{H} \mathbf{x}=\alpha \mathbf{w}^{H} \mathbf{d}+\mathbf{w}^{H} \mathbf{n}$. The SINR at the filter output SINR $R_{\text {out }}$ is:

$$
S I N R_{\text {out }}=\frac{|\alpha|^{2}\left|\mathbf{w}^{H} \mathbf{d}\right|^{2}}{E\left(\mathbf{w}^{H} \mathbf{n} \mathbf{n}^{H} \mathbf{w}\right)}=\frac{|\alpha|^{2}\left|\mathbf{w}^{H} \mathbf{d}\right|^{2}}{\mathbf{w}^{H} \mathbf{\Sigma} \mathbf{w}} .
$$

$S I N R_{\text {out }}$ is maximum when $\mathbf{w}=\mathbf{w}_{\text {opt }}$ and its value is $S I N R_{\max }=|\alpha|^{2} \mathbf{d}^{H} \boldsymbol{\Sigma}^{-1} \mathbf{d}$. The SINR loss, denoted by $\rho$, is the loss of performance when $\mathbf{w}=\hat{\mathbf{w}}\left(\hat{\mathbf{w}}_{S C M}\right.$ or $\left.\hat{\mathbf{w}}_{N S C M}\right)$ :

$$
\rho=\frac{S I N R_{\text {out }}}{S I N R_{\max }}=\frac{\left|\hat{\mathbf{w}}^{H} \mathbf{d}\right|^{2}}{\left(\hat{\mathbf{w}}^{H} \boldsymbol{\Sigma} \hat{\mathbf{w}}\right)\left(\mathbf{d}^{H} \boldsymbol{\Sigma}^{-1} \mathbf{d}\right)} .
$$

From Eq. (9) and (10) the SINR losses, $\rho_{S C M}$ or $\rho_{N S C M}$, of Eq. (12) can be rewritten as:

$$
\rho=\lambda \frac{\left(\mathbf{d}^{H} \hat{\boldsymbol{\Pi}}^{\perp} \mathbf{d}\right)^{2}}{\mathbf{d}^{H} \hat{\boldsymbol{\Pi}}^{\perp} \boldsymbol{\Sigma} \hat{\boldsymbol{\Pi}}^{\perp} \mathbf{d}},
$$

where $\hat{\mathbf{\Pi}}^{\perp}=\hat{\mathbf{\Pi}}_{S C M}^{\perp}$ or $\hat{\mathbf{\Pi}}^{\perp}=\hat{\mathbf{\Pi}}_{N S C M}^{\perp}$. The aim of this section is to compute the mean SINR losses $E\left[\rho_{S C M}\right]$ and $E\left[\rho_{N S C M}\right]$. Results are given in the following proposition.

Proposition 3.1:

$$
E\left[\rho_{S C M}\right]=1-\frac{1}{K} \sum_{i=1}^{r}\left(\frac{E[\tau] \lambda_{i}+\lambda}{E[\tau] \lambda_{i}}\right)^{2}
$$




$$
E\left[\rho_{N S C M}\right]=1-\frac{1}{K} \sum_{i=1}^{r} 2 \frac{E(\tau) \lambda_{i}+\lambda}{\left(\mu_{i}-\mu\right)^{2}} E\left[\frac{\left(\tau \lambda_{i}+\lambda\right) \chi_{i}^{2}(2)}{\left(\sum_{j=1}^{r}\left(\tau \lambda_{j}+\lambda\right) \chi_{j}^{2}(2)+\lambda \chi_{r+2}^{2}(2(m-r))\right)^{2}}\right]
$$

where $\mu_{1}, \ldots, \mu_{r}, \mu$ are the eigenvalues of $\mathbf{R}=E\left[\hat{\mathbf{R}}_{N S C M}\right]$ given in [16] as a function of $\lambda_{1}, \ldots, \lambda_{r}, \lambda$.

Proof: Since all considered estimators have been shown consistent, the SINR loss is evaluated for large $K$ by means of a perturbation analysis [18]. Starting from the perturbations on $\hat{\mathbf{R}}, \hat{\boldsymbol{\Pi}}_{c}$ and $\hat{\boldsymbol{\Pi}}_{c}^{\perp}$, the SINR loss $\rho$ of Eq. (13) is reduced in a compact form thanks to a second order approximation. Let us start by $E\left[\rho_{S C M}\right]$.

First, let us introduce the pseudo-inverse, $\mathbf{M}_{S C M}$, of $\mathbf{S}_{\Sigma}$ (see Eq. (3)):

$$
\mathbf{M}_{S C M}=\sum_{i=1}^{r} \frac{1}{E(\tau) \lambda_{i}} \mathbf{u}_{i} \mathbf{u}_{i}^{H} .
$$

Let $\Delta \boldsymbol{\Sigma}=\hat{\mathbf{R}}_{S C M}-\boldsymbol{\Sigma}$ be the covariance estimation error on $\boldsymbol{\Sigma}$. This estimation error induces an error on the estimates $\hat{\boldsymbol{\Pi}}_{c S C M}$ and $\hat{\boldsymbol{\Pi}}_{c S C M}^{\perp}$. It is shown in [18] that the projector estimates are given up to the second order with respect to $\Delta \Sigma$ by:

$$
\begin{aligned}
\hat{\boldsymbol{\Pi}}_{c S C M} & \approx \boldsymbol{\Pi}_{c}+\delta \boldsymbol{\Pi}_{c}+\delta^{2} \boldsymbol{\Pi}_{c} \\
\hat{\boldsymbol{\Pi}}_{c S C M}^{\perp} & \approx \boldsymbol{\Pi}_{c}^{\perp}-\delta \boldsymbol{\Pi}_{c}-\delta^{2} \boldsymbol{\Pi}_{c}
\end{aligned},
$$

where $\delta \boldsymbol{\Pi}_{c}$ and $\delta^{2} \boldsymbol{\Pi}_{c}$ are equal to:

$$
\begin{aligned}
\delta \boldsymbol{\Pi}_{c} & =\boldsymbol{\Pi}_{c}^{\perp} \Delta \boldsymbol{\Sigma} \mathbf{M}_{S C M}+\mathbf{M}_{S C M} \Delta \boldsymbol{\Sigma} \boldsymbol{\Pi}_{c}^{\perp} \\
\delta^{2} \boldsymbol{\Pi}_{c} & =\boldsymbol{\Pi}_{c}^{\perp} \gamma \mathbf{M}_{S C M}+\mathbf{M}_{S C M} \gamma^{H} \boldsymbol{\Pi}_{c}^{\perp}+\boldsymbol{\Pi}_{c} \boldsymbol{\beta} \boldsymbol{\Pi}_{c}+\boldsymbol{\Pi}_{c}^{\perp} \Delta \boldsymbol{\Sigma} \mathbf{M}_{S C M}^{2} \Delta \boldsymbol{\Sigma} \boldsymbol{\Pi}_{c}^{\perp}
\end{aligned},
$$

where matrices $\gamma$ and $\boldsymbol{\beta}$ are second order terms with respect to $\Delta \boldsymbol{\Sigma}$. In what follows, all equalities are valid up to the second order with respect to $\Delta \boldsymbol{\Sigma}$.

The second-order approximation of the denominator of Eq. (13) yields:

$$
\begin{aligned}
& \mathbf{d}^{H} \hat{\Pi}_{c S C M}^{\perp} \boldsymbol{\Sigma} \hat{\Pi}_{c S C M}^{\perp} \mathbf{d}= \\
& \mathbf{d}^{H} \boldsymbol{\Pi}_{c}^{\perp} \boldsymbol{\Sigma} \boldsymbol{\Pi}_{c}^{\perp} \mathbf{d}-\mathbf{d}^{H} \boldsymbol{\Pi}_{c}^{\perp} \boldsymbol{\Sigma} \delta \boldsymbol{\Pi}_{c} \mathbf{d}-\mathbf{d}^{H} \delta \boldsymbol{\Pi}_{c} \boldsymbol{\Sigma} \boldsymbol{\Pi}_{c}^{\perp} \mathbf{d}+\mathbf{d}^{H} \delta \boldsymbol{\Pi}_{c} \boldsymbol{\Sigma} \delta \boldsymbol{\Pi}_{c} \mathbf{d}-\mathbf{d}^{H} \delta^{2} \boldsymbol{\Pi}_{c} \boldsymbol{\Sigma} \boldsymbol{\Pi}_{c}^{\perp} \mathbf{d}-\mathbf{d}^{H} \boldsymbol{\Pi}_{c}^{\perp} \boldsymbol{\Sigma} \delta^{2} \boldsymbol{\Pi}_{c} \mathbf{d}
\end{aligned}
$$

The first term is equal to $\lambda$ from Eq. (10). The second and the third term are equal to 0 since $\Pi_{c}^{\perp} \Sigma \mathbf{M}_{S C M}=$ $\mathbf{0}$ and $\mathbf{M}_{S C M} \mathbf{d}=\mathbf{0}$. Therefore:

$$
\begin{aligned}
\mathbf{d}^{H} \hat{\boldsymbol{\Pi}}_{c S C M}^{\perp} \boldsymbol{\Sigma} \hat{\boldsymbol{\Pi}}_{c S C M}^{\perp} \mathbf{d} & =\lambda+\mathbf{d}^{H} \delta \boldsymbol{\Pi}_{c} \boldsymbol{\Sigma} \delta \mathbf{\Pi}_{c} \mathbf{d}-2 \lambda \mathbf{d}^{H} \delta^{2} \boldsymbol{\Pi}_{c} \mathbf{d} \\
& =\lambda+\mathbf{d}^{H} \Delta \boldsymbol{\Sigma} \mathbf{M}_{S C M} \boldsymbol{\Sigma} \mathbf{M}_{S C M} \Delta \boldsymbol{\Sigma} \mathbf{d}-2 \lambda \mathbf{d}^{H} \Delta \boldsymbol{\Sigma} \mathbf{M}_{S C M}^{2} \Delta \boldsymbol{\Sigma} \mathbf{d} .
\end{aligned}
$$

We have from Eq. (3) and Eq. (16):

$$
\mathbf{M}_{S C M} \boldsymbol{\Sigma} \mathbf{M}_{S C M}=\mathbf{M}_{S C M}\left(\mathbf{S}_{\Sigma}+\lambda \mathbf{I}_{m}\right) \mathbf{M}_{S C M}=\mathbf{M}_{S C M}+\lambda \mathbf{M}_{S C M}^{2}
$$


From Eq. (21), Eq. (20) becomes:

$$
\mathbf{d}^{H} \hat{\boldsymbol{\Pi}}_{C S C M}^{\perp} \boldsymbol{\Sigma} \hat{\boldsymbol{\Pi}}_{C S C M}^{\perp} \mathbf{d}=\lambda+\mathbf{d}^{H} \Delta \boldsymbol{\Sigma}\left(\mathbf{M}_{S C M}-\lambda \mathbf{M}_{S C M}^{2}\right) \Delta \boldsymbol{\Sigma} \mathbf{d} .
$$

Secondly, let us compute the numerator of Eq. (13). We have:

$$
\mathbf{d}^{H} \hat{\boldsymbol{\Pi}}_{S C M}^{\perp} \mathbf{d}=\mathbf{d}^{H} \boldsymbol{\Pi}_{c}^{\perp} \mathbf{d}-\mathbf{d}^{H} \delta \boldsymbol{\Pi}_{c} \mathbf{d}-\mathbf{d}^{H} \delta^{2} \boldsymbol{\Pi}_{c} \mathbf{d} .
$$

Since $\boldsymbol{\Pi}_{c}^{\perp} \mathbf{d}=\mathbf{d}$ and $\mathbf{M}_{S C M} \mathbf{d}=\mathbf{0}$, Eq. (23) is equivalent to:

$$
\mathbf{d}^{H} \hat{\boldsymbol{\Pi}}_{C S C M}^{\perp} \mathbf{d}=1-\mathbf{d}^{H} \Delta \boldsymbol{\Sigma} \mathbf{M}_{S C M}^{2} \Delta \boldsymbol{\Sigma} \mathbf{d} .
$$

and thus:

$$
\left(\mathbf{d}^{H} \hat{\mathbf{\Pi}}_{c S C M}^{\perp} \mathbf{d}\right)^{2}=1-2 \mathbf{d}^{H} \Delta \mathbf{\Sigma} \mathbf{M}_{S C M}^{2} \Delta \mathbf{\Sigma} \mathbf{d} .
$$

Finally, the second order expression of the SINR loss of Eq. (13) is:

$$
\rho_{S C M}=\lambda \frac{\left(\mathbf{d}^{H} \hat{\boldsymbol{\Pi}}_{c S C M}^{\perp} \mathbf{d}\right)^{2}}{\mathbf{d}^{H} \hat{\boldsymbol{\Pi}}_{c S C M}^{\perp} \boldsymbol{\Sigma} \hat{\boldsymbol{\Pi}}_{c S C M}^{\perp} \mathbf{d}}=1-\mathbf{d}^{H} \Delta \boldsymbol{\Sigma}\left(\frac{1}{\lambda} \mathbf{M}_{S C M}+\mathbf{M}_{S C M}^{2}\right) \Delta \boldsymbol{\Sigma} \mathbf{d} .
$$

As $\mathbf{M}_{S C M} \boldsymbol{\Sigma} \mathbf{d}=\mathbf{0}$ (since $\mathbf{u}_{i}^{H} \mathbf{d} \approx 0$ for $i \leq r$ ), we can substitute $\hat{\mathbf{R}}_{S C M}$ for $\Delta \boldsymbol{\Sigma}$ in Eq. (26):

$$
\rho_{S C M}=1-\mathbf{d}^{H} \hat{\mathbf{R}}_{S C M}\left(\frac{1}{\lambda} \mathbf{M}_{S C M}+\mathbf{M}_{S C M}^{2}\right) \hat{\mathbf{R}}_{S C M} \mathbf{d}=1-\left\|\left(\frac{1}{\lambda} \mathbf{M}_{S C M}+\mathbf{M}_{S C M}^{2}\right)^{1 / 2} \hat{\mathbf{R}}_{S C M} \mathbf{d}\right\|^{2} .
$$

Let us set:

$$
\left(\frac{1}{\lambda} \mathbf{M}_{S C M}+\mathbf{M}_{S C M}^{2}\right)^{1 / 2}=\sum_{i=1}^{r} a_{i} \mathbf{u}_{i} \mathbf{u}_{i}^{H} \text { with } a_{i}=\frac{1}{E[\tau] \lambda_{i}} \sqrt{\frac{E[\tau] \lambda_{i}+\lambda}{\lambda}}
$$

and

$$
\begin{aligned}
& \mathbf{z}_{k}=\left(\frac{1}{\lambda} \mathbf{M}_{S C M}+\mathbf{M}_{S C M}^{2}\right)^{1 / 2} \mathbf{x}_{k} \mathbf{x}_{k}^{H} \mathbf{d} \\
& \mathbf{z}=\left(\frac{1}{\lambda} \mathbf{M}_{S C M}+\mathbf{M}_{S C M}^{2}\right)^{1 / 2} \hat{\mathbf{R}}_{S C M} \mathbf{d}=\frac{1}{K} \sum_{k=1}^{K} \mathbf{z}_{k} .
\end{aligned}
$$

We have:

$$
\rho_{S C M}=1-\|\mathbf{z}\|^{2}
$$

For large $K$, as assumed in this paper, the central limit theorem ensures that $\mathbf{z}$ is Gaussian distributed. Its first and second order moments follow from those of $\mathbf{z}_{k}$ and will be now investigated. The SINR loss distribution will be obtained from these results. The first order moment of $\mathbf{z}_{k}$ is:

$$
E\left(\mathbf{z}_{k}\right)=\left(\frac{1}{\lambda} \mathbf{M}_{S C M}+\mathbf{M}_{S C M}^{2}\right)^{1 / 2} E\left(\mathbf{x}_{k} \mathbf{x}_{k}^{H}\right) \mathbf{d}=\left(\frac{1}{\lambda} \mathbf{M}_{S C M}+\mathbf{M}_{S C M}^{2}\right)^{1 / 2} \mathbf{\Sigma} \mathbf{d}=\mathbf{0},
$$

since $\mathbf{u}_{i}^{H} \mathbf{d} \approx 0$ for $i \leq r$. Let us now compute the second order moments of $\mathbf{z}_{k}$. Let us recall that $\mathbf{x}_{k}=\mathbf{c}_{k}+\mathbf{b}_{k}$ and introduce the following new parameter:

$$
\mathbf{y}_{k}=\left[\mathbf{u}_{1} \ldots \mathbf{u}_{r} \mathbf{d}\right]^{H} \mathbf{x}_{k} .
$$


Conditionally to $\tau_{k}, \mathbf{x}_{k}$ is complex zero-mean Gaussian and its covariance eigensystem is $\tau_{k} \lambda_{1}+\lambda>$ $\tau_{k} \lambda_{2}+\lambda>\ldots>\tau_{k} \lambda_{r}+\lambda>\lambda=\ldots=\lambda$ and $\left\{\mathbf{u}_{1}, \ldots, \mathbf{u}_{r}, \mathbf{u}_{r+1}, \ldots, \mathbf{u}_{m}\right\}$. Consequently, each component of $\mathbf{y}_{k}$, conditionally to $\tau_{k}$, can be written as follows:

$$
\begin{aligned}
\left(\mathbf{y}_{k}\right)_{i} & =\sqrt{\frac{1}{2}\left(\tau_{k} \lambda_{i}+\lambda\right) \chi_{k, i}^{2}(2)} \exp \left(j \theta_{k, i}\right) \quad i=1, \ldots, r \\
\left(\mathbf{y}_{k}\right)_{r+1} & =\sqrt{\frac{1}{2} \lambda \chi_{k, r+1}^{2}(2)} \exp \left(j \theta_{k, r+1}\right)
\end{aligned},
$$

where $\theta_{k, i}$ is uniformly distributed on $[0,2 \pi]$. All random variables are mutually independent. Therefore, we obtain:

$$
\mathbf{z}_{k}=\sum_{i=1}^{r} a_{i}\left(\mathbf{u}_{i}^{H} \mathbf{x}_{k}\right)\left(\mathbf{x}_{k}^{H} \mathbf{d}\right) \mathbf{u}_{i}=\sum_{i=1}^{r} a_{i}\left(\mathbf{y}_{k}\right)_{i}\left(\mathbf{y}_{k}^{H}\right)_{r+1} \mathbf{u}_{i} .
$$

The second order moments of $\mathbf{z}_{k}$ is easily computed from Eqs. (33) and (34): $E\left(\mathbf{z}_{k} \mathbf{z}_{k}^{T}\right)=\mathbf{0}$ and

$$
E\left(\mathbf{z}_{k} \mathbf{z}_{k}^{H}\right)=\sum_{i=1}^{r} \frac{a_{i}^{2}}{4} E\left[\left(\tau_{k} \lambda_{i}+\lambda\right) \lambda \chi_{i}^{2}(2) \chi_{r+1}^{2}(2)\right] \mathbf{u}_{i} \mathbf{u}_{i}^{H}=\sum_{i=1}^{r} a_{i}^{2}\left(E[\tau] \lambda_{i}+\lambda\right) \lambda \mathbf{u}_{i} \mathbf{u}_{i}^{H} .
$$

The SINR loss distribution follows from Eq. (30), (35) and the central limit theorem:

$$
\rho_{S C M}=1-\frac{1}{2 K} \sum_{i=1}^{r}\left(\frac{E[\tau] \lambda_{i}+\lambda}{E[\tau] \lambda_{i}}\right)^{2} \chi_{i}^{2}(2) .
$$

Taking the expectation of Eq. (36) completes the proof for $E\left[\rho_{S C M}\right]$.

The proof for $E\left[\rho_{N S C M}\right]$ is very similar and is omitted.

Remark: In STAP context, the hypothesis of a strong clutter in comparison to the white Gaussian noise is often valid. In this particular case, the SINR losses of proposition 3.1 admit simplified expressions:

$$
\begin{gathered}
E\left[\rho_{S C M}\right] \approx 1-\frac{r}{K} . \\
E\left[\rho_{N S C M}\right] \approx 1-\frac{1}{K} \sum_{i=1}^{r} 2 \frac{E(\tau) \lambda_{i}^{2}}{\mu_{i}^{2}} E\left[\frac{1}{\tau}\right] E\left[\frac{\chi_{i}^{2}(2)}{\left(\sum_{j=1}^{r} \lambda_{j} \chi_{j}^{2}(2)\right)^{2}}\right] .
\end{gathered}
$$

Indeed, we have in the case of a strong clutter $E[\tau] \lambda_{i} \gg \lambda$ for $i=1, \ldots, r$. For $E\left[\rho_{S C M}\right]$ in Eq. (14), Eq. (37) is then easily deduced. Concerning $E\left[\rho_{N S C M}\right]$, it may be shown from [16] that $\mu_{1}, \ldots, \mu_{r} \gg \mu$ when $\lambda$ is small. Expression (38) follows from Eq. (15) for small $\lambda$.

Eq. (37) is similar to the classical result of [9], [11] with Gaussian clutter. This shows that the texture $\tau$ has no influence on the SINR loss $\rho_{S C M}$ in a strong clutter hypothesis. Concerning the behaviour of the SINR loss $\rho_{N S C M}$ of Eq. (38) in a strong clutter context, we notice that $E\left[\rho_{N S C M}\right]$ does depend on the texture distribution. 


\section{NUMERICAL SimUlations}

We consider the following side-looking STAP configuration to check the theoretical SINR losses of proposition 3.1. The number of sensors is $N=8$ and the number of coherent pulses is $M=8$. Angles of arrival are measured with respect to broadside. The center frequency and the bandwidth are respectively equal to $f_{0}=450 \mathrm{MHz}$ and $B=4 \mathrm{MHz}$. The radar velocity is $V=100 \mathrm{~m} / \mathrm{s}$. The inter-element spacing is $d=\frac{c}{2 f_{0}}$ ( $c$ is the speed of light) and the pulse repetition frequency is $f_{r}=600 \mathrm{~Hz}$. The clutter rank is computed from Brennan rule [7]: $r=15(r=15<N M=64)$. The covariance matrix of the Gaussian clutter is simulated thanks to the modelling presented in [1]. To simulate the SIRV clutter, we choose for the texture $\tau$ a Gamma distribution with shape parameter $\nu$ and scale parameter $1 / \nu$ (which results in $E[\tau]=1$ ). The identity matrix is next added to build the covariance matrix $\boldsymbol{\Sigma}$ of Eq. (3). The Clutter to Noise Ratio (CNR) is set to $25 d B$. The clutter ridge spans the angles between $-90^{\circ}$ and $90^{\circ}$ and the speeds between $-100 \mathrm{~m} / \mathrm{s}$ and $100 \mathrm{~m} / \mathrm{s}$. The target velocity is $40 \mathrm{~m} / \mathrm{s}$ with an angle of arrival equal to $-20^{\circ}$. In this configuration, we have $\max _{i \in\{1, r\}}\left(\left|\mathbf{u}_{i}^{H} \mathbf{d}\right|\right)=0.1$. Therefore, we consider a case with the property of non-orthogonality between the target and the clutter subspaces: a simple matched filter is not sufficient to detect the target.

The theoretical LR-SCM and LR-NSCM SINR losses are computed using Eq. (14) and Eq. (15). For the last one, the expectations involving the texture are evaluated by 100000 Monte Carlo trials. The numerical LR-SCM and LR-NSCM SINR losses of Eq. (13) are estimated using 10000 Monte-Carlo trials.

Figure 1 displays the numerical and theoretical SINR losses as a function of $K$ for the LR-STAP filters built from the SCM and NSCM in the case of a LR-SIRV clutter of parameter $\nu=2$ and $\nu=1$. The second case simulates a strong non-homogeneous clutter. For the LR-STAP filter built from the SCM, numerical and theoretical SINR losses are close for both $\nu$. This validates Eq. (14) as well as the approximate expression (37) (texture has no influence on $\rho_{S C M}$ ). For the LR-STAP filter built from the NSCM, numerical and theoretical SINR losses are close for $\nu=2$. When $\nu=1$, the theoretical result is correct for large $K$ (which validates Eq. (15)) but more mitigated for small $K$. Note also as expected in Eq. (38) that the texture distribution has a strong influence on the SINR loss of the LR-STAP filter built from the NSCM (numerical and theoretical) which is not the case for the LR-STAP filter built from the SCM. 

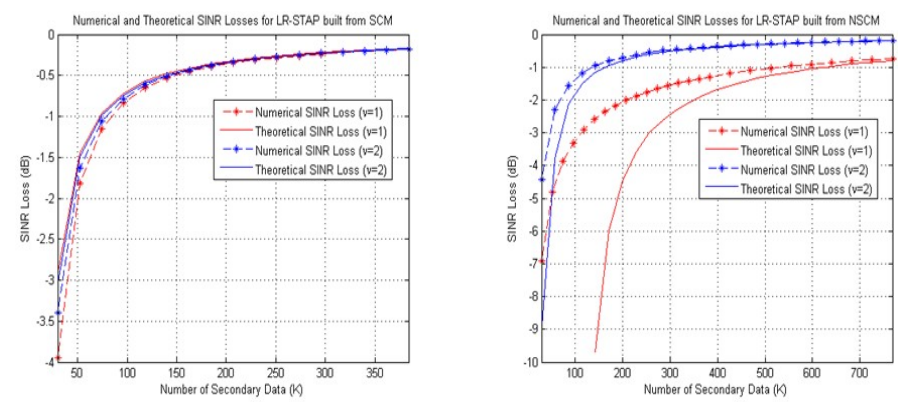

Fig. 1. Theoretical (solid line) and Numerical (dashed line and stars) SINR losses for LR-STAP filters built from the SCM (left) and the NSCM (right) as a function of $K$. The LR-SIRV clutter is simulated by a Gamma distribution of parameter $\nu=1$ (red) and $\nu=2$ (blue)

\section{CONCLUSION}

In this paper, we analyzed the theoretical performance of two LR-STAP filters built from the SCM and the NSCM by deriving SINR loss expressions based on a perturbation analysis. Compared to previous works, disturbance was modeled as the sum of a Low-Rank SIRV clutter and a zero-mean white Gaussian noise. Numerical simulations validated the theoretical formula even in a case of non orthogonality of the target signal with respect to the clutter subspace and allowed to show that the LR-STAP filter built from the SCM has better performance than the one built from the NSCM. For strong clutter to noise ratio, we also proved that the LR-STAP filter built from the SCM leads to the same performance as in Gaussian clutter.

In a future work, we propose to investigate the performance of the LR-STAP filter built from the NSCM for small $\mathrm{K}$ according to the distribution of the texture. It will be also interesting to investigate how the SINR losses will be affected by a wrong estimation of $r$.

\section{REFERENCES}

[1] J. Ward, "Space-time adaptive processing for airborne radar," Tech. Rep., Lincoln Lab., MIT, Lexington, Mass., USA, December 1994.

[2] E. Conte, M.D. Bisceglie, C. Galdi, and G. Ricci, "A procedure for measuring the coherence length of the sea texture," IEEE Trans. Instrum. Meas., vol. 46, no. 4, pp. 836-841, 1997.

[3] J.B. Billingsley, A. Farina, F. Gini, M.V. Greco, and L. Verrazzani, "Statistical analyses of measured radar ground clutter data," IEEE Trans. Aerosp. Electron. Syst., vol. 35, no. 2, pp. 579 - 593, 1999.

[4] K. Yao, "A representation theorem and its applications to spherically invariant random processes," IEEE Trans. Inf. Theory, vol. 19, no. 5, pp. $600-608$, September 1973.

[5] J.B. Billingsley, "Ground clutter measurements for surface-sited radar," Tech. Rep. 780, MIT, February 1993. 
[6] E. Conte, A. De Maio, and C. Galdi, "Statistical analysis of real clutter at different range resolutions," IEEE Trans. Aerosp. Electron. Syst., vol. 40, no. 3, pp. 903 - 918, 2004.

[7] L. E. Brennan and F.M. Staudaher, "Subclutter visibility demonstration," Tech. Rep., RL-TR-92-21, Adaptive Sensors Incorporated, March 1992.

[8] I.S. Reed, J.D. Mallett, and L.E. Brennan, "Rapid convergence rate in adaptive arrays," IEEE Trans. Aerosp. Electron. Syst., vol. AES-10, no. 6, pp. 853 - 863, November 1974.

[9] I. Kirsteins and D. Tufts, “Adaptive detection using a low rank approximation to a data matrix," IEEE Trans. Aerosp. Electron. Syst., vol. 30, pp. 55 - 67, 1994.

[10] A. Haimovich, "The eigencanceler: Adaptive radar by eigenanalysis methods," IEEE Trans. Aerosp. Electron. Syst., vol. 32, no. 2, pp. 532 - 542, April 1996.

[11] A. Haimovich, "Asymptotic distribution of the conditional signal-to-noise ratio in an eigenanalysis-based adaptive array," IEEE Trans. Aerosp. Electron. Syst., vol. 33, pp. 988 - 997, 1997.

[12] C.D. Peckham, A.M. Haimovich, T.F.ï ¡youb, J.S. Goldstein, and I.S. Reed, "Reduced-rank STAP performance analysis," IEEE Trans. Aerosp. Electron. Syst., vol. 36, no. 2, pp. 664 - 676, April 2000.

[13] G. Ginolhac and G. Jourdain, “"Principal Component Inverse" algorithm for detection in the presence of reverberation,” IEEE J. Ocean. Eng., vol. 27, no. 2, pp. 310 - 321, April 2002.

[14] F. Gini, M.V. Greco, and L. Verrazzani, "Detection problem in mixed clutter environment as a Gaussian problem by adaptive pre-processing," Electronics Letters, vol. 31, no. 14, pp. 1189 - 1190, July 1995.

[15] G. Ginolhac, P. Forster, J.P. Ovarlez, and F. Pascal, "Spatio-temporal adaptive detector in non-homogeneous and low-rank clutter," in Proceedings of ICASSP, Taipei, Taïwan, april 2009.

[16] G. Ginolhac, P. Forster, F. Pascal, and J.P. Ovarlez, "Derivation of the bias of the normalized sample covariance matrix in a heterogeneous noise with application to low rank STAP filter," IEEE Trans. Signal Process., vol. 60, no. 2012, pp. 514 - 518, January 2012.

[17] G. Ginolhac and P. Forster, "Performance analysis of a robust low-rank STAP filter in low-rank Gaussian clutter," in Proceedings of ICASSP, Dallas, TX, USA, april 2010.

[18] H. Krim, P. Forster, and J.G. Proakis, "Operator approach to performance analysis of root-music and root-min-norm," IEEE Trans. Signal Process., vol. 40, no. 7, pp. 1687 - 1696, July 1992.

[19] M. Rangaswamy, D.D. Weiner, and A. Ozturk, "Non-Gaussian vector identification using spherically invariant random processes,” IEEE Trans. Aerosp. Electron. Syst., vol. 29, no. 1, pp. 111-124, January 1993.

[20] F. Pascal, P. Forster, J.P. Ovarlez, and P. Larzabal, "On a SIRV-CFAR detector with radar experimentations in implusive noise," in Proceedings of EUSIPCO, Florence, Italy, september 2006.

[21] F. Pascal, Y. Chitour, J.P. Ovarlez, P. Forster, and P. Larzabal, "Existence and characterization of the covariance matrix maximum likelihood estimate in spherically invariant random processes," IEEE Trans. Signal Process., vol. 56, no. 1, pp. $34-48$, January 2008. 